\title{
Aprender sobre la marcha. Prácticas de docencia universitaria en salud pública en la pandemia por el nuevo coronavirus*
}

\author{
Cecilia Molina \\ Universidad Nacional de Cuyo, Mendoza, Argentina \\ ceciliamolina269@gmail.com \\ https://orcid.org/0000-0001-5569-786X \\ María Florencia Lindardelli \\ Universidad Nacional de Cuyo, Mendoza, Argentina \\ linardellimf@gmail.com \\ https://orcid.org/0000-0002-3250-2895
}

\section{RESUMEN}

Este artículo reflexiona sobre la tarea docente en el contexto actual, marcado tanto por la pandemia a causa de la COVID-19 como por las medidas de aislamiento social preventivo y obligatorio dispuestas por el Gobierno nacional argentino desde fines de marzo de 2020. Mediante la estrategia de sistematización de experiencias, se analizan algunas tensiones y desafíos experimentados durante el dictado virtual de la materia Problemática de la Salud, en la carrera de Trabajo Social de la Universidad Nacional de Cuyo (Uncuyo), provincia de Mendoza. Se recuperan algunos debates recientes sobre la educación virtual en contextos universitarios y, a partir de estos,

examinamos de qué manera se vieron afectados los contenidos, el plan de actividades prácticas, los objetivos de aprendizaje, la práctica docente y las experiencias de los y las estudiantes en el contexto modelado por la situación pandémica y el tipo de educación virtual que impuso. Hacia el final abordamos en qué medida pudimos aprovechar las potencialidades que los entornos virtuales reportan y cómo operaron las limitaciones de estos en la experiencia estudiantil y docente que se vivenció durante la pandemia por el nuevo coronavirus.

Palabras clave: salud pública; educación universitaria; educación virtual; COVID-19; aislamiento social.

Cómo citar: Molina, C. y Linardelli, M. F. (2021). Aprender sobre la marcha. Prácticas de docencia universitaria en salud pública y pandemia de coronavirus. Ciencias Sociales y Educación, 10(19), 23-53. https://doi. org/10.22395/csye.v10n19a2

Recibido: 3 de diciembre de 2020.

Aprobado: 20 de febrero de 2021. 


\section{Learn as you go. University Teaching Practices in Public Health and the Coronavirus Pandemic}

\section{ABSTRACT}

This article reflects on the teachers' job in a context influenced by both the pandemic and the preventive social isolation measures applied by the Argentinian National Government from the end of March 2020. Through the strategy of systematization, experiences, tensions, and challenges of virtual classes in the subject Problemática de la Salud (Public Health Problems) in the social work degree at Universidad Nacional de Cuyo (Uncuyo or Cuyo's National University in English) located in the Mendoza province are analyzed. Some recent debates related to virtual education in university contexts are reconside- red and it is from them that we analyze in which way were the contents, the practical activities plan, the learning objectives, the teacher's role, and the experiences of the alumni, affected or influenced by a pandemic context and the type of virtual education that this imposed. In the end, we cover to what extent we were able to take advantage of the potentialities that the virtual learning provides, as well as how its limitations had an impact on the students and teachers' experience through the coronavirus pandemic.

Keywords: public health; higher education; virtual learning; COVID-19; social isolation.

\section{Aprenda conforme você avança. Práticas de ensino universitário em saúde pública e a pandemia de coronavirus}

\section{RESUMO}

Este artigo reflexiona sobre o trabalho docente no contexto marcado pela pandemia do corona vírus quanto pelas medidas de Isolamento Social Preventivo e Obrigatório ordenadas pelo Governo Nacional Argentino desde o final de março de 2020. Por meio da estratégia de sistematização de experiências, são analisadas algumas tensões e desafios vividos durante o ditado virtual da disciplina Problemas de saúde, na carreira do Serviço Social, da Universidade Nacional de Cuyo (Uncuyo), província de Mendoza. Recuperam-se alguns debates recentes sobre a educação virtual em contextos universitários e, a partir deles, examinamos como foram afetados os conteúdos, o plano de atividades práticas, os objetivos de aprendizagem, a prática docente e as experiências dos alunos no contexto moldado pela situação de pandemia e pelo tipo de educação virtual que ela impôs. No final, abordamos até que ponto fomos capazes de aproveitar o potencial que os ambientes virtuais relatam e como suas limitações operaram na experiência do aluno e do professor durante a pandemia do corona vírus.

Palavras-chave: saúde pública; formação universitária; educação virtual; COVID-19; isolamento social. 


\section{Introducción}

En este artículo reflexionamos sobre la tarea docente en el contexto actual, marcado tanto por la pandemia ocasionada por la COVID-19 como por las medidas de aislamiento social preventivo y obligatorio (ASPO) dispuestas por el Gobierno nacional argentino desde fines de marzo de 2020. Analizamos algunas tensiones y desafíos experimentados durante el dictado virtual de la materia Problemática de la Salud que se dicta en la carrera de Trabajo Social de la Universidad Nacional de Cuyo (Uncuyo), en la provincia de Mendoza. Exploramos los obstáculos, ventajas y resultados del dictado de una materia que, por su pertenencia al campo temático de la salud pública, transita tópicos ubicados en el centro de las preocupaciones sociales de los últimos meses.

¿Quién podía imaginar, hacia fines de 2019, que epidemiólogos/as serían consultados a diario por periodistas y políticos/as? Tampoco podía preverse que el índice de camas hospitalarias por habitante sería un indicador al que los gobernantes y la ciudadanía empezarían a prestarle atención. Al comenzar el año, estaba lejos de nuestras proyecciones que algunos de los contenidos más replicados en redes sociales serían indicadores de morbilidad y mortalidad de un virus desconocido, ni que Twitter y Facebook se convertirían en espacios de debate de políticas sanitarias. Nuestra materia comenzaba su dictado anual en un escenario complejo, en el que la salud adquirió centralidad.

En el plano subjetivo, esta circunstancia nos enfrentaba con la incertidumbre del "no saber" o del solo "saber que no sabemos" (Schord Landman, 2020). Además, como resultado de las medidas preventivas dispuestas, no solo se ponían "en suspenso" nuestra vida cotidiana, agendas y hábitos, sino que se abría una amplia brecha entre nuestras experiencias y el horizonte de expectativas (Scholten et al., 2020).

La materia Problemática de la Salud es una asignatura cuatrimestral que se dicta en el primer período de cuarto año de la carrera de Trabajo Social. Entre sus contenidos aborda las distintas construcciones teóricas en torno al proceso de salud/enfermedad/cuidados y sus relaciones con las políticas sociales, la participación social y el ejercicio de derechos. Apuntamos a que los estudiantes puedan incorporarlos de manera crítica y creativa en diferentes escenarios de intervención y no solo el de los servicios de salud. Desde 2011, la cátedra utiliza el entorno virtual de la universidad (www.uncuvirtual.uncuyo.edu.ar) como una herramienta complementaria a las clases presenciales, los horarios de consulta y las reuniones periódicas con docentes tutores/as, que son las estrategias pedagógicas de mayor peso. Justamente, fue esta distribución la que se invirtió por completo con el inicio del ASPO desde mediados de marzo de 2020. 
Desde esa fecha hasta mayo el país estuvo afectado de manera homogénea por el aislamiento que fue dictado y renovado a través de sucesivos decretos presidenciales. Entre mayo y junio, varias provincias argentinas finalizaron el aislamiento estricto y progresivamente ingresaron a un régimen de "distanciamiento social" con protocolos sanitarios. En agosto, el notorio aumento de casos obligó a varias jurisdicciones a volver al aislamiento, incluida la provincia de Mendoza. Durante todo este período, la Uncuyo - al igual que otras casas de altos estudios - permaneció cerrada, al tiempo que resolvió adaptar el dictado y la evaluación de todas sus carreras a modalidad virtual. Por este motivo, el curso de Problemática de la Salud, que sucedió entre marzo y junio del año 2020, fue completamente virtual.

Al comenzar el dictado, al menos tres asuntos nos ocupaban como equipo docente para afrontar el singular contexto. En primer lugar, pensábamos que la pandemia constituía un escenario patente para el análisis de los principales ejes temáticos de la asignatura, por lo que incluimos esta "novedad epidemiológica" entre los contenidos y actividades habituales. En segundo lugar, anticipábamos los desafíos que impondría el dictado virtual de clases, en buena medida por las brechas tecnológicas en nuestro país y porque muchos estudiantes de la carrera de trabajo social de la Uncuyo utilizan los insumos tecnológicos ofrecidos por la universidad y que ahora están vedados por las medidas de aislamiento. Por último, subyacía una gran preocupación sobre la posibilidad de acompañar y sostener los procesos de aprendizaje en este contexto. El desarrollo del cursado evidenció que una parte de nuestras anticipaciones se concretaban en la experiencia, mientras que otras eran opacadas en el encuentro efectivo con los y las estudiantes, sus realidades e inquietudes.

En este marco, al finalizar el dictado de la materia nos propusimos reflexionar sobre el proceso vivido, en particular sobre los desafíos y tensiones de la tarea docente en salud pública en el contexto de la epidemia global del coronavirus y las medidas de aislamiento. El objetivo de este artículo es presentar los resultados de la sistematización de esa experiencia educativa. Además del registro de la tarea docente realizado durante el dictado de la asignatura, analizamos las percepciones de estudiantes y docentes, que fueron recogidas mediante cuestionarios virtuales aplicados en septiembre y octubre de 2020.

El escrito se organiza en cuatro secciones. Primero se presentan las nociones teóricas centrales de la materia que orientan el proceso de enseñanza y aprendizaje en salud pública y algunos debates recientes sobre educación virtual. Segundo, se detalla el encuadre metodológico. Luego, se exponen los resultados de la sistematización y se analizan alternativamente las experiencias y opiniones de docentes y estudiantes. Hacia el final se ponderan los resultados 
y aprendizajes de la enseñanza virtual durante la pandemia de coronavirus y las medidas de ASPO. Concluimos con reflexiones y preguntas sobre las novedades, persistencias y desafíos en los procesos de enseñanza-aprendizaje transitados.

\section{Marco teórico y conceptual}

Como punto de partida, recuperamos algunas nociones clave que permiten comprender los contenidos y estrategias pedagógicas de la materia Problemática de la Salud.

La asignatura postula que las categorías de salud-enfermedad son "ventanas abiertas para la comprensión de las acciones humanas, de las relaciones entre los individuos y la sociedad, de las instituciones y sus mecanismos de dirección y control" (Minayo, 2009, p. 197). Los contenidos abordados se distancian del paradigma biomédico socialmente dominante, por cuanto este limita el horizonte de posibilidades de intervención de las disciplinas sociales en el campo sanitario. La perspectiva biomédica mantiene una mirada individual, asistencialista y ahistórica que suele ocultar los condicionantes históricos, sociales y económicos que operan en los procesos de salud-enfermedad (Menéndez, 1990). Además, desconoce que los modos de vivir y enfermar de sujetos y colectivos están atravesados por dinámicas globales que se expresan de manera desigual en los territorios (Molina Jaramillo, 2018).

En contraposición a esa perspectiva, desde principios del siglo pasado las ciencias sociales aportan evidencia empírica que permite dar cuenta de que la salud y la enfermedad no se reducen a una evidencia biológica, orgánica, natural, individual y objetiva. Más bien, su comprensión por parte de sujetos y grupos sociales es una realidad construida, como lo son las respuestas sociales para enfrentar los padecimientos, la vida y la muerte (Evans, Barer y Marmor, 1996; Ramos, 2006).

Por ello, entendemos la salud como un proceso socialmente determinado por las circunstancias en que las personas nacen, crecen, viven, trabajan y envejecen (López Arellano, Escudero y Dary Carmona, 2008). "Esas circunstancias son el resultado de la distribución del dinero, el poder y los recursos a nivel mundial, nacional y local, que depende a su vez de las políticas adoptadas" (OMS, 2008). La determinación de la salud, por tanto, incluye la consideración de modelos de desarrollo, políticas económicas y sociales y sistemas políticos. Cuando las relaciones entre esas fuerzas y sistemas son inequitativas, dan lugar a inequidades en salud (OPS, 2017).

En sintonía con estos planteos, el programa de la asignatura despliega nociones y herramientas vinculadas con la promoción de la salud (OMS, 1986), 
propuestas participativas de educación popular y de comunicación para la salud (Petracci, 2012) y el enfoque de "salud en todas las políticas" (Ståhl et al., 2006). Para desarrollar capacidades orientadas a identificar e intervenir en problemas y respuestas sociosanitarias, la materia se vale de herramientas de la epidemiología y la demografía, de procedimientos para analizar la situación de salud en organizaciones o poblaciones pequeñas (Pría Barros, 2006) y de mapeos de activos sociales para la salud comunitaria (García et al., 2019).

El plan de actividades prácticas se orienta y recupera aspectos de la "educación permanente en salud". Esta perspectiva, inspirada en la pedagogía crítica y el constructivismo, concibe que

\begin{abstract}
una información o experiencia pedagógica solamente produce sentido cuando dialoga con toda la acumulación anterior que las personas traen consigo. Partir de las incomodidades, dialogar con lo acumulado y producir sentido son las claves para el aprendizaje significativo en el campo de la salud. (Merhy et al., 2006, p. 153)
\end{abstract}

Inscribimos los objetivos de aprendizaje en el enfoque de capacidades, que se distancia de la formación por competencias y es dominante en la enseñanza universitaria argentina desde fines del siglo pasado. De acuerdo con Vilafranca y Buxarrais (2009), el foco en las capacidades traza como meta de la educación superior fomentar la agencia, autonomía y empoderamiento de los y las estudiantes (Citado en Boni Aristizábal, Lozano y Walker, 2010, p. 129). Dicho horizonte requiere promover "la reflexión crítica y la discusión argumentativa [...] sobre el mundo y la vida que llevamos" (Boni Aristizábal, Lozano y Walker, 2010, p. 129).

En función de los lineamientos teóricos y pedagógicos expuestos, la asignatura incorporó en 2011 los entornos virtuales a las estrategias de enseñanza-aprendizaje, con un rol complementario a las actividades presenciales. La enseñanza virtual ha ganado terreno en la educación universitaria en la última década, especialmente en las instancias de posgrado. Algunas de las ventajas más obvias que reporta se relacionan, por un lado, con la posibilidad de acercarse a personas residentes en territorios distantes del lugar donde se dicta la capacitación y, por otro, con el dinamismo temporal que posibilita a quienes participan en la capacitación (la asincronía de las clases).

Más allá de estas posibilidades, en los últimos años se debate sobre otras cualidades de esta modalidad frente al aprendizaje tradicional, cada vez más puesto en cuestión por docentes y estudiantes. Se sostiene que la interacción virtual en el campo educativo permite experimentar vínculos pedagógicos más horizontales y democráticos, al tiempo que favorece la construcción colaborativa de conocimiento en el marco de espacios socioeducativos expandidos (Díez-Gutiérrez y Díaz-Nafría, 2018; Burbules, 2014). 
Estos rasgos contrastan, a criterio de los y las autores/as mencionados, con características muy arraigadas en el aprendizaje tradicional, como la rígida separación entre contextos educativos formales y no formales, el distanciamiento con los asuntos y problemas de la vida cotidiana de las personas implicadas, la preeminencia de la lectoescritura como medio de aprendizaje y la reproducción de la lógica que entiende las disciplinas como compartimentos estancos. Ellos advierten que las herramientas de los entornos tecnológicos posibilitan nuevas experiencias educativas, pero también que internet y las redes sociales "no solo están transformando el proceso de aprendizaje y enseñanza, sino que también están afectando profundamente al modo en el que nos conectamos y comunicamos" (Díez-Gutiérrez y Díaz-Nafría, 2018, p. 56). Se afirma que en los entornos digitales se aprende de otra manera, porque se construye y comparte conocimiento de otra forma. Esto da lugar a nuevas perspectivas sobre el aprendizaje "concebido ahora como social, distribuido y situado" (Fueyo Gutiérrez, Braga Blanco, Fano Méndez, 2015, p. 120).

Hay autores/as que asocian la educación virtual con una "mayor ubicuidad" en los procesos de aprendizaje, por cuanto las actividades educativas se integran al flujo de la vida y las relaciones diarias (Burbules, 2014). A la vez, los y las educadores/as, familiarizados con formas más tradicionales de enseñar se ven impelidos/as a ensamblar las redes sociales con los entornos sociales, comunicativos y vitales de las personas participantes (Díez-Gutiérrez y DíazNafría, 2018).

En otros aspectos se observa menor posibilidad de innovación. Pese a la creciente utilización de redes sociales en los últimos quince años, su introducción en la educación es conflictiva. En particular, entre estudiantes universitarios/as hay un uso intensivo de las redes y permanecen conectados/as prácticamente durante todo el día. Sin embargo, la aplicación y utilización académica de las redes es escasa y, cuando sucede, es por iniciativa propia antes que por incentivo del profesorado, que raramente incorpora estas herramientas en la enseñanza (Fueyo Gutiérrez, Braga Blanco, Fano Méndez, 2015, p. 122).

De acuerdo con las fuentes, las redes tienen un enorme potencial para el aprendizaje, lo que incentiva a preguntarnos cómo utilizar este potencial sin atentar contra aquello que las hace valiosas y atractivas para los y las estudiantes. Además, recalcan que entre los educadores y las educadoras existen temores vinculados con que la utilización de este entorno permita que lo personal, informal o emocional se introduzcan excesivamente en el aula y cuestionen la visión moderna del conocimiento escolar como representante de la racionalidad universal. Quizás por ello la utilización de redes sociales ha estado plagada 
de temores y desconfianza o, por lo menos, de una actitud defensiva entre los educadores y las educadoras.

Las perspectivas conceptuales aquí reseñadas nos conducen a plantear algunas inquietudes que atraviesan los resultados compartidos a continuación. En primer lugar, examinamos de qué manera se vieron modelados/afectados los contenidos, el plan de actividades prácticas, los objetivos de aprendizaje, la práctica docente y las experiencias de estudiantes en el escenario planteado por la situación pandémica. En segundo lugar, nos preguntamos si, empujados/ as al completo desarrollo virtual de la materia, pudimos aprovechar las potencialidades que los entornos virtuales reportan y cómo operaron las limitaciones propias de la virtualidad en la experiencia estudiantil y docente realizada durante la pandemia.

\section{Estrategia metodológica}

Este artículo presenta los resultados de la sistematización de una experiencia docente. La sistematización de experiencias es una metodología ampliamente utilizada en el campo educativo. De acuerdo con Jara (2013), consiste en la interpretación crítica de una o varias experiencias, que son reconstruidas para dilucidar la lógica y los sentidos subyacentes en el proceso vivido, los diversos factores que intervinieron y su relación. La sistematización se propone producir conocimientos y aprendizajes significativos que posibiliten "apropiarse críticamente de las experiencias vividas, comprenderlas teóricamente y orientarlas hacia el futuro con una perspectiva transformadora" (Jara, 2013, p. 78).

La sistematización comenzó con reuniones periódicas de una parte de las docentes, en las que reflexionamos sobre los desafíos y tensiones vivenciados durante el dictado de la materia. En esos encuentros también analizamos los exámenes finales, en particular, una pregunta que solicitaba desarrollar dos nociones o capacidades adquiridas durante el curso para comprender mejor o intervenir en la situación de emergencia y confinamiento social. Luego de este proceso concluimos que era necesario ampliar nuestras fuentes de información para realizar la sistematización, por lo que elaboramos instrumentos de recolección de datos para recuperar las percepciones del estudiantado y del equipo docente.

Por un lado, confeccionamos una guía de preguntas abiertas que fue respondida por el conjunto de los y las docentes de la materia ${ }^{1}$ a través de WhatsApp y correo electrónico. Las preguntas se focalizaron en tres temáticas: i) la influencia

En 2020 el equipo de cátedra estuvo integrado por la profesora titular, una adjunta, una jefa de trabajos prácticos y dos auxiliares de docencia, en condición de rentadas. También participaron tres docentes adscriptos. La adscripción docente es una figura frecuente en las universidades nacionales argentinas, a través de la cual se realiza una formación que habilita a ingresar a la carrera docente (sin ser excluyente) e implica el cumplimiento de un conjunto de tareas acotadas que se realizan ad-honorem. 
de la coyuntura sanitaria y el dictado virtual de la materia en la tarea docente y en los procesos de aprendizaje; ii) las adaptaciones/modificaciones personales, horarias, vinculares, tecnológicas que debieron realizar por el singular contexto; iii) las diferencias de la experiencia de 2020 con respecto a años anteriores.

Por otro lado, desarrollamos un cuestionario en la plataforma Google Forms, que fue administrado a estudiantes al finalizar el curso. El cuestionario se estructuró en veinticuatro preguntas (que admitían respuestas cerradas y abiertas) e indagó por las condiciones en que desarrollaron el curso, la experiencia con los tutores y las tutoras, así como con las opiniones generales sobre los contenidos y la modalidad virtual. El instrumento se puso a disposición en octubre de 2020, después de finalizar el curso, para quienes regularizaron la materia. La difusión se realizó a través de los tutores y las tutoras, especialmente por WhatsApp. En el transcurso de una semana se obtuvieron treinta y seis respuestas, lo que representa el 37,5\% de los inscriptos/as en 2020.

El material recabado se analizó con diferentes técnicas según el tipo de información obtenida. Los datos surgidos de las respuestas docentes fueron abordados siguiendo el análisis temático y categorial propio de las técnicas cualitativas (Minayo, 2009). Los resultados del cuestionario dirigido a los y las estudiantes se trabajaron, mediante el análisis de contenido, en las preguntas de respuesta abierta. El tratamiento estadístico-descriptivo de los datos resultantes de las preguntas de respuesta cerrada se circunscribió al cálculo de las frecuencias y de los porcentajes que representaban respecto del total de respuestas.

\section{Resultados}

\section{La experiencia desde el punto de vista del equipo docente}

Durante el primer semestre de 2020, el plantel de la cátedra estuvo integrado por cinco docentes rentadas - con dedicaciones horarias diversas, aunque en su mayoría se trata de cargos simples- y tres adscriptos/as ad honorem. Las primeras son la profesora titular, una adjunta, dos auxiliares de docencia y una profesional que, por extensión de funciones en el Hospital de la Uncuyo, cumple parte de su carga horaria en docencia. En cuanto a la procedencia disciplinar, seis de las profesoras son trabajadoras sociales, uno es psicólogo, y otro es licenciado en Ciencias Políticas. Esta dotación de docentes, de carácter excepcional, fue clave para afrontar el proceso de enseñanza- aprendizaje en el contexto de emergencia sanitaria. En palabras de una profesora: "el hecho - un tanto fortuito, porque no es lo habitual- de contar con un equipo de ocho personas para desarrollar la materia permitió atenuar la mayor carga 
de trabajo y lograr los resultados que tuvimos" (profesora titular, comunicación personal, 13 de noviembre de 2020).

El dictado de la materia comenzó prácticamente en simultáneo con el decreto del ASPO. Los docentes comenzamos el ciclo con pesimismo, ansiedad e incertidumbre. Con independencia de las posiciones en la cátedra y en la facultad, advertimos que enfrentábamos dificultades pedagógicas excepcionales, entre ellas i) las limitaciones de los y las estudiantes para acceder a la conectividad y al equipamiento informático; ii) probables mermas en la cantidad de inscriptos/as; y iii) deserción durante el cursado por falta de actividades presenciales, de interacción cara a cara en las clases y espacios de consulta.

No obstante, y sin estar ajenas/os a temores del conjunto, algunos/as docentes avistamos que el nuevo contexto también podía dar lugar a oportunidades. Esto se debe a que, por un lado, como la materia se dicta en el primer semestre, se dispondría de más energías y más tiempo — por coincidir con la etapa más estricta del ASPO- para generar los contenidos íntegramente virtuales. Por el otro, se vislumbró que con la pandemia por el nuevo coronavirus, la problemática de la salud podría adquirir una nueva centralidad en la formación de los estudiantes de Trabajo Social. En este sentido dijo uno de los participantes:

estaba en el centro de la escena, como pocas veces podía yo recordar, la discusión sobre las políticas de salud, las estrategias de prevención, los límites de la biomedicina, términos epidemiológicos poblaban los medios de comunicación y las redes sociales. (Auxiliar de docencia, comunicación personal, 15 de noviembre de 2020)

Más allá de estas presunciones, al finalizar el cuatrimestre pudimos revisar cuán acertadas o alejadas de la experiencia efectiva se encontraban estas anticipaciones, y reflexionar sobre la tarea realizada.

\section{Influencias de la coyuntura sanitaria y la virtualidad en el dictado de la materia}

En primer lugar, desde el equipo docente identificamos que la coyuntura (pandemia y ASPO) tuvo, entre otras consecuencias, nuevas "disponibilidades" en estudiantes, producto del aislamiento, la inédita valoración social que adquirió la salud pública y las adaptaciones que realizó el equipo de cátedra al nuevo escenario. En términos generales, coincidimos en que las/os estudiantes dispusieron de más tiempo para dedicar al curso. Además, advertimos que, por la edad promedio de la mayoría, el estudiantado está familiarizado con la comprensión y el manejo de los formatos audiovisuales y las plataformas virtuales utilizadas para desarrollar clases, trabajos prácticos e instancias de evaluación. 
En segundo lugar, durante el cursado notamos que la pandemia y el ASPO supusieron una atención nueva sobre ejes clave de la asignatura que no suelen ser puestas en valor. Por ejemplo, las respuestas del sistema de salud frente a una emergencia, las brechas sociales en el acceso a la salud y a la tecnología, así como la vinculación entre actores e instituciones cuyas políticas deben converger para enfrentar los determinantes de la salud.

Además, percibimos que la coyuntura modificó modalidades habituales de relación con estudiantes, y que esos cambios operaron como oportunidades para el desarrollo de la materia. Hubo coincidencias respecto de que, en relación con cohortes anteriores, tuvimos una mayor disponibilidad y apelación simultánea a dispositivos como el aula virtual, los grupos de WhatsApp, los teléfonos y correos personales para compartir materiales, facilitar el acceso a contenidos y disipar dudas: "El intercambiar consultas y preguntas en el espacio de los grupos de WhatsApp para la tutoría, con un número reducido de estudiantes, le aportó una horizontalidad y cotidianidad a la comunicación, que no había experimentado antes" (profesora adjunta, comunicación personal, 19 de noviembre de 2020)

Emergió también en las apreciaciones docentes una mayor receptividad a situaciones específicas de estudiantes y más imbricación de la docencia con la vida cotidiana de cada uno/a. En el escenario de emergencia, la docencia implicó mucha creatividad, necesidad de producir materiales audiovisuales nuevos, mayor disposición horaria e involucramientos que excedieron, de lejos, lo que se esperaría de la dedicación de un/a profesor/a en condiciones habituales. Con respecto a esto, uno de los auxiliares afirma: "tuve que acostumbrarme a establecer el contacto de otro modo, y a encontrar la manera de lograr que los/ las estudiantes accedan a los conceptos de la forma más ilustrativa posible" (auxiliar de docencia, comunicación personal, 16 de noviembre de 2020).

Otra modificación significativa de las tareas docentes se vinculó con nuevos cuidados y mayores exigencias en la preparación de las clases virtuales. Además, el imperativo de adecuar contenidos disparó la inquietud de poner en diálogo los propios con los de otras materias del currículo. Con respecto a esto, los y las docentes y auxiliares reflexionaron lo siguiente.

Este formato permite que podamos revisar con mayor detenimiento cada contenido que se les proporciona a los estudiantes e inclusive revisar hasta los conceptos que se expresan verbalmente, porque todo queda registrado digitalmente, cosa que de forma presencial no es posible. (Docente adscripta, comunicación personal, 14 de noviembre de 2020)

clases mucho más guionadas, más "practicadas", más conceptuales y precisas que, además, tienen la posibilidad de ser escuchadas repetidamente en caso de dudas. (Auxiliar de docencia, comunicación personal, 16 de noviembre de 2020) 
ajustamos la bibliografía, hice algo que no había hecho en años anteriores, que fue dialogar con profesores de otras materias que dictan contenidos afines y leer su bibliografía para hacer una síntesis que facilitara que los estudiantes pudiesen recuperarlas y recrearlas en el contexto de la problemática de la salud. (Profesora adjunta, comunicación personal, 19 de noviembre de 2020)

El imperativo de seleccionar contenidos, priorizar materiales de estudio, reemplazar las clases expositivas por dispositivos audiovisuales breves y concisos y realizar devoluciones orales o escritas sintéticas y adaptables a las posibilidades del estudiantado habilitaron poner en cuestión ciertos mitos y dogmas presentes en la formación de grado de trabajadores sociales. Advertíamos estos mitos y dogmas antes de la epidemia global, con cierto malestar, sin que ello redundara - hasta ahora - en un cuestionamiento profundo y un cambio en nuestra dinámica de trabajo y de relacionamiento con el resto de la carrera.

Parte de las adecuaciones que se identificaron como necesarias derivan de la mayor mediación tecnológica que involucra desarrollar una materia de manera virtual. Estos acomodamientos demandaron desplegar nuevas capacidades y recursos, e interpelaron los modos aprendidos de ejercer la docencia antes del ASPO. Como ha sido indicado por investigaciones recientes (Martínez-Garcés y GarcésFuenmayor, 2020), en el contexto de la virtualidad, movilizada por la pandemia de coronavirus, la creación de contenido digital es una de las "competencias digitales" que se han mostrado más débiles entre docentes. También el manejo de la seguridad en entornos informáticos (protección de datos personales, por ejemplo) fue identificado como una habilidad poco desarrollada por educadores/as. Además de las dificultades mencionadas, el equipo docente reconoció que el reemplazo obligado de las clases y consultas presenciales por medios virtuales exigió una nueva y mayor carga laboral, cuya contracara fue que resultaron más amigables para el estudiantado. En este sentido, expresan lo siguiente:

Tuve que aprender rápidamente a manejar los distintos dispositivos, plataformas que no formaban parte de nuestra cotidianidad, grabar videos para que los y las estudiantes nos conocieran y para introducir y sintetizar cada unidad o bien modificar el pedido de gráficos, mapas conceptuales, cuadros y otros detalles que pudieran dificultar la resolución de los prácticos. (Jefa de trabajos prácticos, comunicación personal, 21 de noviembre de 2020)

Me generó bastante incertidumbre la falta de ese feedback inmediato que permite la presencialidad. [...] hubo una participación más activa, quizás porque el modo de comunicarse en estas circunstancias (a través de WhatsApp, por ejemplo) puede resultarles más cómodo y habitual para interactuar. (Docente adscripto, comunicación personal, 20 de noviembre de 2020).

En sintonía con lo expresado por los y las estudiantes, uno de los grandes desafíos que conllevó la enseñanza virtual durante el aislamiento fue la modificación en la administración de los tiempos. Los docentes y las docentes 
compartimos que se requirió de mayor plasticidad en un contexto que también modificó la dinámica de trabajo del equipo de cátedra: "ser flexibles con las fechas y los plazos de trabajo y para responder dudas a cualquier hora porque los alumnos contaban con teléfonos y mail personales" (auxiliar de docencia, comunicación personal, 15 de noviembre de 2020); "hubo un aumento de horas para cubrir la demanda de los estudiantes y disminución de los encuentros de equipo docente" (auxiliar de docencia, comunicación personal, 16 de noviembre de 2020); "disponer de algunas horas más para el acompañamiento de los estudiantes en el grupo de WhatsApp, generar espacios de consulta individual o con el uso de videollamada" (docente adscripto, comunicación personal, 15 de noviembre de 2020).

La nueva situación de enseñanza-aprendizaje aparejó adecuaciones en la vida cotidiana personal y familiar. Requirió la gestión de recursos tecnológicos compartidos con familiares, la transformación de ciertos ambientes hogareños y la adaptación de los horarios domésticos. En este sentido, afirmaron en las entrevistas:

fue necesario adaptar y organizar los horarios hogareños para el uso de la única computadora familiar. (Docente adscripta, comunicación personal, 14 de noviembre de 2020)

generar un espacio cómodo en casa para trabajar, adquirir auriculares y ordenar una carpeta digital para hacer control de planillas y seguimiento de entrega de los trabajos prácticos entregados, descargar material de consulta para tenerlo a mano para los estudiantes. Tomar tiempo para realizar entregas y devoluciones de trabajos valorando la composición realizada por lxs [sic] estudiantes. (Auxiliar de docencia, comunicación personal, 15 de noviembre de 2020)

la difícil o imposible manera de hacer cortes entre los dos ámbitos, la exposición de estar trabajando en presencia del resto de los integrantes del grupo familiar conviviente, la sobredependencia de acceso a internet y de los elementos electrónicos de comunicación, entre otros ajustes. (Jefa de trabajos prácticos, comunicación personal, 21 de noviembre de 2020)

Cabe destacar que la mayor parte del equipo trabajó en servicios de salud en simultaneidad con la tarea en la universidad. Esta doble inserción laboral fue claramente advertida como factor de sobrecarga:

como mantuve y hasta incrementé el resto de mi carga laboral -en salud y académica, porque, por ejemplo, varios tesistas rezagados aprovecharon el momento para cerrar sus tesis-, terminé el cuatrimestre con mayor desgaste que en ediciones anteriores. (Profesora adjunta, comunicación personal, 19 de noviembre de 2020)

trabajando un tiempo 7 por 7 y en otro tiempo 14 por 14 , con los chicos en mi casa y un esposo haciendo home office, fue difícil. También se nos rompió una compu [sic] en abril, lo que significó un esfuerzo de organización familiar/laboral. (Docente adscripta, comunicación personal, 14 de noviembre de 2020) 
Como puede observarse, múltiples transformaciones coyunturales modelaron la experiencia docente durante la pandemia. Algunas se vincularon con las limitaciones impuestas por las medidas de aislamiento. Otras, con la acelerada adecuación a la virtualidad y una parte relevante de esta experiencia, fueron marcadas por las transformaciones en la cotidianidad y la carga de trabajo. El conjunto de estos aspectos incidió, a su vez, en los resultados, como veremos a continuación.

\section{Valoración global de la tarea docente}

En general, la evaluación de la tarea docente en el contexto de la pandemia y el ASPO es favorable. Este juicio se asienta, en parte, en los resultados en términos de sostenimiento del curso y alta proporción de estudiantes que regularizaron la materia en la primera parte del año y la aprobaron en las mesas 2020, en comparación con años anteriores. Esta apreciación se hace sin desconocer el esfuerzo que supuso afrontar la tarea:

dimos clase al inicio de la pandemia, con todo lo que significaba, siendo pioneros de alguna manera por encontrarnos en el primer cuatrimestre. Por habernos encontrado con esta realidad un poco de golpe y casi sin aviso, creo que estuvo muy bien. (Docente adscripto, comunicación personal, 20 de noviembre de 2020)

El desgaste y cansancio real percibido por docentes es directamente proporcional con los beneficios reales experimentados por la mayoría de los/las estudiantes. (Profesora titular, comunicación personal, 13 de noviembre de 2020)

Los y las integrantes de la cátedra estimamos que estos logros fueron posibles por el compromiso de docentes y estudiantes, quienes procuraron que "a través de la virtualidad, se pudiera garantizar el vínculo pedagógico". Destacamos la disponibilidad para contactarnos con los y las estudiantes por diversos medios y en diferentes momentos, aunque advertimos que esta situación deberá regularse y limitarse a futuro. También subrayamos la atención y tramitación de necesidades singulares de los y las estudiantes como recurso para sostener el curso. Asimismo, identificamos que el hecho de no trasladarse hasta la facultad abrió la oportunidad, entre estudiantes, de organizarse para relacionarse con los insumos que se produjeron en el momento en que estaban dispuestos a prestar atención y, para los y las docentes, de una mejor preparación de las clases. El conjunto de estos elementos, a criterio del equipo, contribuyó a garantizar el derecho a la educación:

Deberíamos analizar si no tener que pagar transporte, no poder realizar prácticamente ninguna actividad (quédate en casa), pérdida de trabajo o trabajo en casa, no importar las distancias ya sea en otro departamento o provincia, llevaron al resultado de menor desgranamiento durante el dictado, mayor participación y poder terminar el ciclo habiendo rendido el final. (Auxiliar de docencia, comunicación personal, 16 de noviembre de 2020) 
Los educadores y las educadoras compartimos que la capacidad para afrontar la nueva situación de enseñanza-aprendizaje descansó en buena medida en una serie de fortalezas del equipo. Entre ellas, se destacan la experiencia adquirida en el uso de la plataforma virtual como soporte de la enseñanza presencial, que se desarrolla desde hace casi diez años. También implicó la diversidad de perfiles docentes, la complementación de roles y una mayor implicación frente al nuevo desafío. Otra fortaleza identificada fue la capacidad de adaptarse y de construir nuevas respuestas y acuerdos, tomando como oportunidad los interrogantes que disparó una pandemia:

la flexibilidad de pensar los contenidos y ejercicios prácticos para que los estudiantes pudieran realizar una reflexión en su entorno más cercano sobre el impacto y cumplimiento de las medidas del aislamiento social preventivo y obligatorio. (Auxiliar de docencia, comunicación personal, 16 de noviembre de 2020)

Ahora bien, además de valorar las fortalezas, se acuerda que el contexto generó mayores exigencias personales, profesionales y académicas:

una invasión de momentos de descanso, que fue bastante agotadora. (Jefa de trabajos prácticos, comunicación personal, 21 de noviembre de 2020)

dadas las condiciones en que trabajamos, creo que buena parte de los logros se asientan en una intensificación del trabajo docente. (Docente adscripto, comunicación personal, 15 de noviembre de 2020)

lo que más me costó fue lidiar con la sensación de que no estaba formada/preparada para el trabajo en entornos virtuales. Ese aprender sobre la marcha, en medio de una gran incertidumbre del contexto, me resultó muy demandante y por momentos agotador. (Auxiliar de docencia, comunicación personal, 15 de noviembre de 2020)

mucho, mucho estrés, especialmente para generar material grabado de las clases expositivas. (Auxiliar de docencia, comunicación, 16 de noviembre de 2020)

resolver las falencias tecnológicas que tenía en mi hogar para poder realizar las tareas docentes: desde mandar a arreglar mi computadora personal, que tenía el micrófono roto, pedir prestada otra para remplazarla, hasta cambiar el servicio de internet que era insuficiente para la demanda de trabajo virtual. (Docente adscripta, comunicación personal, 14 de noviembre de 2020)

\section{La experiencia desde el punto de vista de las y los estudiantes}

En 2020 se inscribieron ciento dieciocho estudiantes para cursar la materia. Del total de inscriptos/as, la notable mayoría fueron mujeres y solo cursaron nueve varones, lo que coincide con el perfil feminizado de la carrera. El $43 \%$ tiene menos de veintidós años; el 34 , entre veintidós y veintisiete años, y el $33 \%$ restante supera esas edades. Estos datos marcan un porcentaje superior al 70 \% y son jóvenes o adultos jóvenes. Como indican los datos expresados, el perfil 
mayoritario de los y las cursantes es de mujeres jóvenes que cursan la carrera de Trabajo Social.

La mayor parte de la información que presentamos sobre los y las estudiantes fue recabada mediante el cuestionario virtual. Entre quienes respondieron el cuestionario, la mayoría son estudiantes de la carrera de Trabajo Social, mientras que solo dos pertenecen a otras careras (Sociología y Tecnicatura en Políticas Públicas), y seleccionaron la asignatura como electiva. Aunque el índice de respuesta fue acotado, consideramos que permite identificar algunas tendencias relevantes que analizamos en los siguientes apartados.

\section{Condiciones de cursado durante la pandemia y las medidas de ASPO}

Debido a que el dictado de la materia fue completamente virtual, los y las estudiantes debieron utilizar los insumos tecnológicos disponibles en sus hogares para afrontar el cursado. Al respecto, hallamos que más de la mitad de quienes respondieron el cuestionario utilizó más de un tipo de dispositivo para acceder a las clases virtuales. Entre las opciones que combinaron, el 71 \% apeló al celular, el $57 \%$ a un portátil o a una tableta y el $43 \%$ a una computadora de escritorio. Solo tres personas dispusieron únicamente de celular. La mitad de los y las estudiantes debieron compartir con familiares, amigos o compañeros de trabajo los dispositivos que utilizaban para conectarse. Cerca del $45 \%$ de los y las estudiantes consultados/as calificó su conectividad a internet como regular y solo dos personas manifestaron tener graves límites de conectividad ${ }^{2}$.

Dadas las notorias modificaciones en la cotidianidad por la pandemia, nos interesó indagar en las tareas extraacadémicas que los y las estudiantes sostuvieron mientras cursaban Problemática de la Salud. Primero, encontramos que la proporción que trabajó de manera remunerada (presencial o virtual) durante el cursado y la de quienes no lo hicieron fue similar ${ }^{3}$. Segundo, un 20 \% manifestó haber visto aumentada su carga de trabajo remunerado respecto de la situación previa al aislamiento. También indagamos en la carga de trabajo no remunerado

2 El acceso limitado a conectividad que reportan los y las estudiantes consultados coincide con lo recabado en un estudio más amplio realizada por el Servicio de Orientación de nuestra facultad, de acuerdo con la cual el $32 \%$ de las y los estudiantes de Trabajo Social solo acceden a internet a través de datos móviles. Este porcentaje es de $14 \%$ en la carrera de Comunicación Social, en la que $63 \%$ de los y las estudiantes manifiestan que pueden conectarse a través de wifi (Volman, Raimondi y Maturano, 2020, p. 6).

3 El trabajo de Volman, Raimondi y Maturano (2020, p. 5) profundiza en las posibilidades de avance académico en el contexto de la pandemia cuando la trayectoria educativa convive con la trayectoria laboral y condiciona la situación socioeconómica. En ese reporte se marca que algo más de la mitad de los estudiantes de la facultad manifiesta que ha continuado trabajando durante la cuarentena: el 57 \% realiza teletrabajo. En este grupo se expresan las dificultades relativas a la convivencia de actividades académicas, laborales y familiares, lo que complejiza la administración y uso del tiempo que se destina al desarrollo de actividades académicas. Por su parte, una alta proporción de quienes no trabajaron manifiesta profunda preocupación por su situación económica, debido a que gran parte de ellas y ellos han perdido el trabajo por la crisis generada por la pandemia. 
y hallamos que el $47 \%$ de los y las estudiantes aumentaron el tiempo dedicado a las tareas domésticas y de cuidado. Finalmente, consultamos por las actividades de militancia o trabajo barrial. Esta pregunta arrojó que la gran mayoría (77 \%) no realizó actividades de este tipo durante el curso, mientras que el $11 \%$ afirmó que se incrementó el tiempo dedicado estas actividades respecto de la dedicación habitual que mantenían antes del confinamiento.

Para completar el panorama de condiciones del curso, indagamos la cantidad de horas semanales dedicadas a la materia durante el cuatrimestre. Un $48 \%$ dedicó entre cuatro y siete horas, el 38 \% mantuvo una dedicación de entre ocho y diez horas, mientras que una proporción menor mantuvo dedicaciones por debajo (6 \%) o por encima de los otros segmentos (6\%) (figura 1).

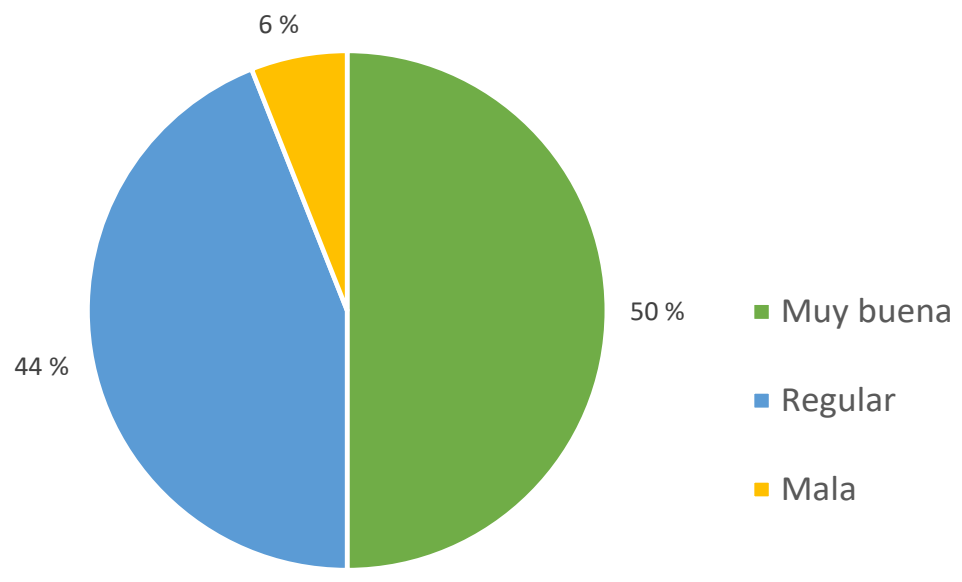

Figura 1. Calidad de la conexión a internet durante el curso

Fuente: elaboración propia con base en las respuestas de los estudiantes al cuestionario virtual autoadministrado.

Los datos hasta aquí expuestos señalan algunos asuntos relevantes. En primer lugar vemos que, respecto de la disponibilidad de insumos tecnológicos, en todos los casos tuvieron algún dispositivo para conectarse y solo dos personas calificaron su conexión a internet como mala. Sin embargo, el hecho de que la mitad del estudiantado no dispusiera de un dispositivo a tiempo completo (dado que debía compartirlo con otras personas) y que no tuviese una conectividad a internet óptima, habla del esfuerzo que requirió para ellos y ellas sostener su participación en el curso. También resulta relevante que el dispositivo más utilizado fuese el teléfono celular, instrumento con menores funciones y comodidades para visualizar clases, producir textos y leer bibliografía extensa. Una consulta más amplia realizada por el servicio de orientación de nuestra facultad advierte que las limitaciones con los dispositivos complejizan el proceso de 
enseñanza-aprendizaje en varios frentes. En este sentido, Volman, Raimondi y Maturano (2020) afirman:

la lectura de textos desde la pantalla reducida del celular, las complicaciones para la realización de trabajos y actividades solicitadas por las cátedras, especialmente la elaboración de textos en formato de Word y Power Point, a lo que se suman otras dificultades referidas a la escasa memoria disponible en los dispositivos, en su mayoría antiguos o compartidos con otros miembros de la familia, lo que dificultad la descarga de aplicaciones y recursos solicitados por las cátedras. (p. 6)

En segundo lugar, la información recabada sobre la carga de trabajo remunerado y no remunerado indica dos datos llamativos: i) el 20 \% vio incrementada su carga de trabajo remunerado habitual y ii) el aumento del trabajo doméstico y de cuidados durante la pandemia afectó casi al $50 \%$ de las y los estudiantes. Estos datos son consistentes con el informe publicado recientemente por la Dirección Nacional de Economía, Igualdad y Género (2020), en el que se indica que el trabajo doméstico y de cuidados no remunerado es una de las actividades que aumentó su carga notablemente durante la pandemia. Como veremos más adelante, particularmente el cuidado de niños y niñas aparece como una situación que tensionó el cumplimiento de las exigencias académicas. Sobre este punto, las variables generacionales y de género se entrecruzan para explicar la mayor carga doméstica (y la menor disponibilidad de tiempo para otras actividades) de las mujeres que son madres. En todo caso, ambos datos muestran un aumento de cargas de trabajo que acompañaron a una proporción relevante de los y las estudiantes durante el cursado.

\section{Dificultades y oportunidades del cursado virtual durante la pandemia y el ASPO}

Un tema que nos interesó particularmente tiene que ver con las dificultades y oportunidades identificadas por los y las estudiantes para leer, cumplir con las consignas, interactuar con tutores y rendir exámenes en el nuevo contexto. En cuanto a las dificultades, de manera predominante, encontramos dos asuntos en las respuestas que coinciden con los datos presentados en la sección anterior. Por un lado, se evidenció la falta de tiempo en el marco de cambios de la carga de trabajo cotidiana. Por otro lado, se perciben problemas en la disponibilidad de conectividad y de dispositivos para trabajar de manera virtual. Algunas expresiones revelan la vivencia de estas dificultades:

Tuve mucho trabajo, más de lo normal, además de mis hijas las 24 horas en casa. (Estudiante mujer de Trabajo Social, comunicación personal, 10 de octubre de 2020) ${ }^{4}$

Cada una de las expresiones textuales que se presentan en este apartado corresponden a diferentes estudiantes y fueron relevadas mediante un cuestionario virtual cuyas características se detallaron en la sección metodológica de este artículo. 
Tuve cierta dificultad al entregar los prácticos a tiempo, ya que por momentos estaba muy complicada por temas de superposición de horarios con el trabajo. (Estudiante mujer de Trabajo Social, comunicación personal, 15 de octubre de 2020)

el hecho de tener que compartir la compu [sic] fue al principio un obstáculo, hasta que organizamos los horarios de uso. (Estudiante mujer de Trabajo Social, comunicación personal, 2 de octubre de 2020)

Aunque aludido con menos frecuencia, el cambio en las formas de enseñanzaaprendizaje que impuso la virtualidad fue otra de las dificultades referidas. Se destacan malestares por la ausencia de espacios compartidos con pares; la tensión entre los requerimientos de concentración propios del estudio y sus condiciones domésticas; y una percepción de incertidumbre en cuanto a la forma en que se desarrollarían las evaluaciones.

Estar muchas horas frente a la pantalla y sin estar con otras compañeras fue lo más dificultoso o cansador. (Estudiante mujer de Trabajo Social, comunicación personal, 15 de octubre de 2020)

En mi casa no tengo un espacio específico para estudiar, lo hago en el living, y eso a veces me presentaba dificultades para concentrarme. (Estudiante mujer de Trabajo Social, comunicación personal, 4 de octubre de 2020)

También se mencionan una serie de problemas que pueden asociarse a malestares emergentes o padecimientos mentales que surgieron o se profundizaron en este contexto inédito de confinamiento social. En este sentido, se afirma en el cuestionario realizado:

En el actual contexto ha sido dificultosa la cuestión del encierro y, al trabajar en la salud, me costaba concentrarme a la hora de leer textos. (Estudiante mujer de Trabajo Social, comunicación personal, 10 de octubre de 2020)

Dolores físicos, agotamiento mental, angustias, entre otros. (Estudiante de Trabajo Social de intercambio, comunicación personal, 8 de octubre de 2020)

Fui víctima de violencia durante el cursado. (Estudiante de Trabajo Social, mujer, comunicación personal, 4 de octubre de 2020)

Otros problemas identificados por los/as estudiantes se relacionan de modo más directo con el programa de la materia, las exigencias curriculares y la plataforma virtual utilizada. Esta última es una herramienta que fue creada para la implementación de ofertas educativas a distancia y que paulatinamente fue incorporada en la enseñanza presencial para actividades puntuales. En muchos casos, antes de la pandemia, funcionaba más como un repositorio de bibliografía que como un ámbito de encuentro entre docentes y estudiantes. Aunque nuestra materia incorpora desde hace varios años el uso de esta plataforma entre las estrategias pedagógicas, su utilización intensiva durante el dictado 
virtual supuso algunas tensiones, vinculadas con ciertas características de este recurso, que resulta poca dinámica e intuitiva. Algunas de las dificultades con la plataforma pueden ser identificadas en las palabras de los/as estudiantes:

La Uncuvirtual resulta complicada para enviar trabajos, ya que no siempre se envían correctamente los archivos y más de una vez tuvimos que terminar enviando los TP por mail. Pero todo lo demás de la plataforma funciona bastante bien. (Estudiante mujer de Sociología, comunicación personal, 10 de octubre de 2020)

Algunos power [sic] no se escuchaban, pero después lo fueron solucionados subiendo al Drive los power [sic] y audios. (Estudiante mujer de Trabajo Social, , comunicación personal, 6 de octubre de 2020)

No estaba acostumbrada a trabajar con tantas bibliografías. (Estudiante mujer de Trabajo Social, comunicación personal, 15 de octubre de 2020)

Pese a tales tensiones, el $75 \%$ de los y las estudiantes manifestaron que siempre pudo acceder con facilidad a la cátedra virtual para descargar bibliografía y visualizar las clases en línea. El 18 \% tuvo acceso casi siempre, y el $7 \%$ pocas veces.

En cuanto a las oportunidades para transitar la materia en el contexto de ASPO, en general, se alude a la posibilidad de realizar una gestión más libre de los tiempos y, en algunos casos, se destaca la mayor disponibilidad horaria en situaciones en las que estudiar "no compite" con otras opciones (laborales, sociales o recreativas, por ejemplo).

Este año dejé de trabajar, entonces pude dedicar tiempo completo a la facultad y al cursado, sumado a que tengo el privilegio de contar con computadora propia e internet en mi casa. (Estudiante mujer de Sociología, comunicación personal, 9 de octubre de 2020)

Tomo como una ventaja las clases online... el poder ver las clases cuando yo quiera también me pareció una excelente oportunidad, porque al ser asincrónicas, yo podía verlas en un momento en el que me sintiera bien y con ganas de hacerlo. Eso aumenta mi productividad y creo que me ayudó a entender bien la materia y poder dedicarle el tiempo que requería. (Estudiante mujer de Trabajo Social, comunicación personal, 1 de octubre de 2020)

También se destacó con frecuencia la ventaja que supuso "ahorrarse" el tiempo de traslado hasta la facultad:

El cursado virtual fue una gran ventaja, ya que vivo muy lejos de la facultad, y el no tener que trasladarme hasta allá me hizo ganar tiempo para estudiar en mi casa, acomodando mis tiempos a los horarios de mi trabajo. (Estudiante mujer de Trabajo Social, comunicación personal, 3 de octubre de 2020) 
Algunos estudiantes señalaron que, incluso, al disponer individualmente dispositivos para el cursado virtual, lograron mejor organización y mayor productividad que en el cursado presencial: "Tuve la posibilidad de poder contar con un espacio para poder estudiar y leer tranquila y también poder acceder a los dispositivos como celular y compu [sic]. Creo que tener esas dos partes fue fundamental para el cursado" (estudiante mujer de Trabajo Social, comunicación personal, 15 de octubre de 2020).

Nos interesa detenernos en las valoraciones sobre la modalidad de resolución de los trabajos prácticos. Esta instancia es central en el dictado de la materia y demanda diversas instancias de intercambio de estudiantes entre sí y con los y las docentes. Durante 2020 propusimos realizar esta tarea de manera individual o en parejas (69 \% optó por esta última modalidad) y las actividades se modificaron parcialmente para su realización de manera virtual. Como se aprecia en la tabla 1, la flexibilidad y la libertad en la administración de los tiempos, la disponibilidad de espacios físicos para estudiar y la conectividad operaron como ventaja para un segmento de estudiantes y para otros como desventaja para la realización de los prácticos.

Tabla 1. Percepciones de estudiantes respecto de la modalidad virtual de resolución de trabajos prácticos

\begin{tabular}{ll}
\hline \multicolumn{1}{c}{ Desventajas } & \multicolumn{1}{c}{ Ventajas } \\
\hline $\begin{array}{l}\text { Se desdibujan los límites de las tareas co- } \\
\text { tidianas en el día a día. }\end{array}$ & $\begin{array}{l}\text { Adaptar el estudio a los horarios no labo- } \\
\text { rales }\end{array}$ \\
$\begin{array}{l}\text { No poder juntarse de manera presencial } \\
\text { en grupo y sacarse dudas y debatir, cara a }\end{array}$ & $\begin{array}{l}\text { Tener acompañamiento grupal y de los pro- } \\
\text { fesores. }\end{array}$ \\
$\begin{array}{l}\text { cara, en vivo, con ejemplos, con profesores } \\
\text { y compañeras. }\end{array}$ & Tener más tiempo por no estar trabajando. \\
$\begin{array}{l}\text { Dificultad para establecer tiempo específi- } \\
\text { co para dedicar a los prácticos. }\end{array}$ & $\begin{array}{l}\text { Ce pudo dedicar más tiempo a los prácticos. } \\
\text { dialogaba y acordaban horarios para elabo- }\end{array}$ \\
$\begin{array}{l}\text { No tener acceso adecuado a internet. } \\
\text { rener diferentes horarios de conectividad }\end{array}$ & $\begin{array}{l}\text { La posibilidad de elegir con quién hacerlos. } \\
\text { con las compañeras del grupo de los mó- } \\
\text { dulos prácticos dificulta una comunica- }\end{array}$ \\
$\begin{array}{l}\text { ción fluida. } \\
\text { Poder analizar contenidos, transcribir ideas }\end{array}$ \\
$\begin{array}{l}\text { No disponer de un lugar de estudio adecua- } \\
\text { do y tener dificultades para concentrarme. }\end{array}$ & $\begin{array}{l}\text { Familiarizarse con herramientas como Goo- } \\
\text { gle Drive, Meet, etc. }\end{array}$ \\
Muchas veces no hay motivación. & Mayor concentración. \\
\hline
\end{tabular}

Fuente: elaboración propia. 
También se perciben como oportunidades algunas de las estrategias pedagógicas desarrolladas desde la cátedra y emergen reflexiones que ponen en valor cierta capacidad de adaptación docente al nuevo entorno. Un sentimiento compartido entre muchos y muchas estudiantes es que el desarrollo de los prácticos se vio favorecido por las decisiones del equipo docente de trabajar en grupos más pequeños que los que se promovían de manera presencial y el énfasis en el acompañamiento sostenido por parte de los tutores y las tutoras:

Siempre tuvimos oportunidad de cumplir con las consignas y tengo entendido que la cátedra fue flexible con compañeres [sic] que no tenían las mismas posibilidades de cumplir con los trabajos o si tenían situaciones particulares. (Estudiante mujer de Trabajo Social, comunicación personal, 10 de octubre de 2020)

otras materias enviaban mucho material y trabajos sin tener en cuenta el contexto en el que estamos. Entonces eso me presentaba dificultades para leer los textos a tiempo y poder hacer los trabajos con tranquilidad. [...]. Por eso valoro mucho lo que la cátedra Problemática de la Salud hizo de disminuir la bibliografía y asignarnos tutoras para poder tener un mejor cursado y acompañamiento. (Estudiante hombre de Trabajo Social, comunicación personal, 3 de octubre de 2020)

los hacías con tiempo y luego solo los enviabas y siempre obtuvimos rápidas devoluciones y, al ser en comisiones, fue muy importante y resultó agradable la materia. (Estudiante mujer de intercambio internacional, comunicación personal, 15 de octubre de 2020)

lo organizada que estaba la cátedra, que permitió que los desafíos de la virtualidad no se sintieran. Se pudo acceder de forma más rápida a la producciónentrega-corrección. (Estudiante mujer de Trabajo Social, comunicación personal, 11 de octubre de 2020)

El canal de comunicación más habitual entre estudiantes y tutores fue WhatsApp, en la modalidad de grupos (60\% de las respuestas) o la mensajería privada de esta red social ( $25 \%$ de las respuestas). Esta tendencia coincide con los datos de un estudio exploratorio, realizado en Mendoza recientemente, que señala que entre los recursos tecnológicos más utilizados por docentes durante el confinamiento se destaca WhatsApp, por cuanto resulta una tecnología de uso masivo que no demanda mayor pericia para su utilización (Expósito y Marsollier, 2020). En menor medida, las comunicaciones fueron por correos electrónicos privados (8 \% de las respuestas), mientras que la mensajería de la plataforma Uncuvirtual no se consignó como medio significativo para los intercambios. La cantidad de tiempo destinado por tutores y tutoras para consultas y otros intercambios se consideró suficiente para el $97 \%$ de los estudiantes que respondieron el cuestionario.

Al indagar por la valoración acerca de la calidad del acompañamiento, las respuestas fueron altamente favorables. Entre los aspectos más positivos, se 
destacan la posibilidad de comunicarse con los y las tutores por diferentes canales, la predisposición para responder rápidamente a las dudas en diferentes horarios y la "claridad" y "precisión" de las devoluciones escritas. Se reiteran expresiones de satisfacción por la disponibilidad y calidez en el trato dispensado en las tutorías, se sintieron "acompañadas", "apoyadas" y "motivadas".

Me sorprendió bastante el trabajo tan personalizado, por la cantidad que éramos cursando. (Estudiante mujer de Trabajo Social, comunicación personal, 9 de octubre de 2020)

Mantuve una interacción constante con la profe y así pude finalizar satisfactoriamente mi cursado. (Estudiante mujer de Trabajo Social, comunicación personal, 1 de octubre de 2020)

El acompañamiento de wasap [sic] con los tutores nos acercó, nos facilitó el entendimiento de los contenidos y nos mantuvo informados en todo momento. (Estudiante mujer de Trabajo Social, comunicación personal, 13 de octubre de 2020)

Subían las clases, explicaban los prácticos a través de videos, la tutora nos resolvía dudas a través de audios de wsp [sic] y el examen fue adecuado al contexto. Gracias a todo eso, logré entender muy bien los conceptos. (Estudiante hombre de Trabajo Social, comunicación personal, 3 de octubre de 2020)

Al igual que puede advertirse en las respuestas docentes, la flexibilidad de los y las docentes destaca como un elemento central en el sostenimiento de la estrategia pedagógica. Este dato se evidencia también en el hecho de que el canal de comunicación más recuente fuese WhatsApp, una red poco estructurada e informal que permite enviar diversos tipos de mensajes y contenidos con rapidez y fluidez. El contrapunto de esta flexibilidad docente es la percepción de mayor carga y extensión de la jornada laboral enunciado en las respuestas del equipo de cátedra.

\section{Relevancia de los contenidos de la materia en la coyuntura sanitaria}

Una parte importante del cuestionario compartido con los y las estudiantes se orientó a captar sus intereses respecto de los contenidos del programa. En particular, nos importaba corroborar si la coyuntura sanitaria había incidido en la disposición hacia ciertos temas de la materia.

Los y las estudiantes mencionaron que los contenidos que despertaron mayor interés son, en primer lugar, las desigualdades sexo-genéricas como determinantes de la salud (54\%). Siguen en orden de interés los contenidos sobre determinantes sociales de la salud, la estrategia de salud en todas las políticas y la promoción de la salud (43\%). Detrás se ubican el trabajo intersectorial e interdisciplinario en salud (34 \%) y los modelos teóricos del proceso salud/enfermedad (26 \%). Los temas que menos menciones merecieron fueron: 
i) atención primaria de la salud, ii) intervención profesional en el campo de la salud, iii) políticas de salud en los estados neoliberales y en los estados sociales de derecho, iv) el sistema de salud argentino y de Mendoza, v) marcos normativos para la intervención profesional en salud, epidemiología y vi) análisis de situación de salud.

Esta priorización de contenidos se relaciona con inquietudes de índole muy diversa. Prevalece entre las respuestas estudiantiles la puesta en valor de aquellos ejes temáticos que se refieren a áreas de conocimiento "que no se abordan en ninguna otra materia", que se interpreta que "guardan directa relación" con las intervenciones profesionales y que contribuyen a disipar dudas y prejuicios con las competencias del trabajo social en el campo de la salud. En este sentido, uno de los estudiantes afirmó: "Elegí los contenidos que me ayudaron a comprender más los posibles abordajes que puede realizar el trabajo social" (Estudiante mujer de trabajo social, comunicación personal, 6 de octubre de 2020).

Un segundo grupo de justificaciones frecuentes son sumamente genéricas. Remiten a formulaciones vinculadas a los discursos dominantes sobre la formación y alcances de la disciplina. En esta línea se expresa: "Elegí los contenidos que más me han aportado al pensamiento crítico" (estudiante mujer de trabajo social, comunicación personal, 11 de octubre) o bien que "la representación social del trabajo social en salud sigue en proceso de deconstrucción, es importante que la intervención profesional sea interdisciplinaria. Apostar por una perspectiva de derechos, más integral, en función del enfoque de salud en todas las políticas" (estudiante mujer de trabajo social, comunicación personal, 14 de octubre de 2020).

En menor medida, se priorizan ciertos contenidos porque se entiende que aportan claves para entender la situación de pandemia o bien para analizar del sistema de servicios de salud y la política de salud como capítulo de las políticas sociales. En este sentido, afirmó una estudiante:

No imaginaba la relevancia de las políticas públicas respecto a salud y la transversalidad de esta respecto al resto de las políticas. Como así también la manera en que afectan los determinantes sociales la salud-enfermedad- cuidado. Trabajarlo en un contexto como el actual me dejó mucho aprendizaje. (Estudiante mujer de trabajo social, Comunicación personal, 10 de octubre de 2020)

La mayor parte de las respuestas no asocian la relevancia de los contenidos al contexto inmediato de la pandemia. Aquí surge un contrapunto con las presunciones docentes que anticipaban una mayor incidencia del contexto sanitario en el interés que suscitarían ciertos contenidos. 
Tanto en los exámenes como en las respuestas del cuestionario advertimos una tendencia a ponderar los temas abordados en la materia de acuerdo con valoraciones amplias que asocian, de maneras un tanto ambiguas, categorías teóricas de gran alcance y abstracción con conceptos de escala más reducida y de mayor concreción. Este vínculo pareciera carecer de la mediación de herramientas analíticas de alcance intermedio. Por ejemplo, se ligan las nociones de intersectorialidad e interdisciplina con el "pensamiento crítico" y la "garantía de los derechos humanos". Nuestra experiencia como docentes de la carrera nos permite entrever que no se trata de una situación que atañe exclusivamente a la materia Problemática de la Salud. De hecho, existen trabajos previos que han analizado este tema y señalan que se trata de un rasgo de ciertas producciones teóricas de la disciplina. En este sentido, afirma Lobos (2020):

Muchas producciones en Trabajo Social se caracterizan por definir poco, partir de supuestos que no se aclaran - lo que produce una notable autohipnosis-, cubrir ciertos conceptos de las ciencias sociales con un tono moral, apelar al pensamiento binario y abusar de algunos bellos términos como sujeto, "otro", autonomía, emancipación, liberación y proyecto ético-político. (p. 4, cursivas en el original)

Aunque no pretendemos profundizar sobre la recepción de teorías críticas que debate el autor, consideramos que estamos ante un asunto a seguir explorando. Resulta un hallazgo relevante que, en un escenario plagado de referencias contextuales para analizar desde las herramientas teóricas y metodológicas de la asignatura, tengan preeminencia las lecturas extremadamente abstractas, como las que aluden a la "deconstrucción del trabajo social en salud", o excesivamente concretas, como las que ponderan "haber comprendido mejor las incumbencias de la disciplina". Lo que llama nuestra atención es el poco interés por instrumentos conceptuales y prácticos que posibilitan realizar análisis situados del campo sanitario, las políticas de salud y la coyuntura epidemiológica.

Lo anterior se vincula, a nuestro juicio, con ciertas dificultades que percibimos desde hace años en la formación, más que con el escenario de la virtualidad. En nombre de las teorías críticas, es habitual que se disocien los marcos conceptuales de la explicación de los problemas sociales singulares, así como del diseño y la implementación de estrategias para afrontarlos. Tales usos de las teorías empobrecen la capacidad que pueden tener ciertos modelos explicativos para preguntarnos y abordar de manera fundada las realidades en las que intervenimos. Desde nuestra perspectiva, esta falencia puede interpretarse como una reacción, poco acertada, al empirismo del que el trabajo social busca despojarse frecuentemente. Cabe señalar que es una tendencia que se expresa tanto entre docentes como estudiantes, en quienes opera una cierta subestimación de las mediaciones conceptuales y la puesta en valor de herramientas al servicio de intervenciones fundadas y específicas. 


\section{Algunos resultados y aprendizajes de la enseñanza virtual durante la pandemia de coronavirus y las medidas de ASPO}

Un asunto relevante para comprender lo que implicó esta experiencia radica en analizar los resultados académicos. Para esto abordaremos dos temáticas. Por una parte, compararemos la cantidad de inscriptos y aprobados en la materia en 2020 y en años anteriores. Por otra, exploraremos las apreciaciones de estudiantes y docentes sobre los aprendizajes alcanzados.

Una manera posible de dimensionar eventuales impactos de la enseñanza virtual fue recuperar información sistematizada por la cátedra sobre la relación inscriptos y aprobados en los años previos a la pandemia y contrastarla con los resultados del 2020. Este ejercicio permite visualizar que, contra las anticipaciones iniciales, la virtualidad y la pandemia no supuso mayores niveles de deserción ni peores indicadores de rendimiento académico.

Cotejando los datos advertimos que el promedio de inscriptos en el período 2012-2019 fue de noventa y seis estudiantes por año. Sobre el total de los inscriptos, en ese mismo período, promocionaron la materia en promedio $72 \%$ de los/as estudiantes. En 2020 se inscribieron para cursar ciento dieciocho estudiantes y noventa y seis (81 \%) la aprobaron desarrollando cuatro trabajos prácticos, un examen parcial y un cuestionario integrador final (en julio) o un coloquio (en mesas de setiembre y octubre). Si comparamos este indicador con años anteriores, finalizaron exitosamente la materia $10 \%$ más que el promedio histórico de los últimos nueve años. Los estudiantes que durante 2020 quedaron en condición de libres (veintidós) no presentaron prácticos ni se contactaron a través de la cátedra virtual. Esto quiere decir que se trata de personas que se inscribieron, pero no efectivizaron el cursado, situación semejante a la de años anteriores.

En cuanto a los aprendizajes a lograr, de acuerdo con el programa de la asignatura, estos se orientan, por una parte, a incorporar nuevos conceptos y recuperar nociones desarrolladas en materias de los años anteriores que aportan a la comprensión de la problemática de la salud. Por otra, se propuso desarrollar capacidades que tienen relación directa con el "saber hacer" del trabajo social en diferentes campos de intervención, no solo el de los servicios de salud.

Cuando les pedimos a los y las estudiantes que expresaran qué aprendizajes consideraban alcanzados, hallamos una diversidad de situaciones. Hay aprendizajes sobre los cuales existe mayor cantidad de estudiantes que perciben haberlos alcanzado satisfactoriamente. Es el caso de desarrollar ideas propias y fundadas en torno a los contenidos de la asignatura; profundizar en una problemática de salud de particular interés e identificar estrategias de prevención y de promoción de la salud relacionadas con problemas de salud prevalentes. Luego 
encontramos un grupo de aprendizajes cuyo logro es considerado satisfactorio por la mitad del grupo de estudiantes, mientras que la otra mitad manifiesta haber logrado poco o nada este aprendizaje. Es el caso de familiarizarse con las diferentes concepciones del proceso salud/enfermedad; identificar ejes para poder analizar políticas o programas de salud; y ejercitar la capacidad de comunicar de manera escrita las producciones y los hallazgos. Finalmente, los aprendizajes en los que identifican mayores dificultades refieren, por un lado, a conocer indicadores demográficos, sanitarios y socioeconómicos para realizar diagnósticos de salud en organizaciones o territorios y, por otro, interpretar esos indicadores y ponerlos al servicio de estrategias de salud comunitaria.

Estas percepciones estudiantiles coinciden con las dificultades identificadas por el equipo docente. Entendemos que la pandemia favoreció la puesta en valor del enfoque de los determinantes sociales, la comprensión de los sistemas de atención de la salud y de los factores que posibilitan o restringen el derecho a la salud de los ciudadanos y las ciudadanas, debido al contexto en que la salud, las políticas sanitarias y sus operadores adquirieron un protagonismo insólito. También creemos que la referencia constante a las causas y efectos de la epidemia global en la vida cotidiana, que circuló en redes sociales y en los espacios de intercambio entre docentes y estudiantes, colaboró en la adquisición de la capacidad para analizar y evaluar programas y respuestas sociales.

Aunque no podemos afirmarlo categóricamente, tanto en las evaluaciones como en los trabajos prácticos hubo un grupo de habilidades en las que los y las estudiantes mostraron mayores problemas. Se trata de las capacidades para vincular información epidemiológica, demográfica y producciones científicoacadémicas del campo de la salud pública con diagnósticos, diseños y ejecuciones de estrategias de salud poblacional, desde organizaciones o en territorios delimitados. La mayor dificultad que identificamos es una especie de divorcio entre los objetivos de aprendizaje que tienden a desarrollar un pensamiento conceptual y analítico y las capacidades más relacionadas al "saber hacer" que se propone a los estudiantes, como interpretar indicadores sociosanitarios que operen como insumos para el diseño y la ejecución de estrategias poblacionales o adquirir herramientas para trabajar en equipos interdisciplinarios y en organizaciones.

Estos aprendizajes que, a criterio estudiantil y docente, suscitaron mayores obstáculos, también denotaron mayor complejidad en años anteriores. Esto quiere decir que esta dificultad no ha variado sustancialmente durante el dictado virtual de la materia, aunque se modificaron con particular atención las estrategias pedagógicas y las actividades prácticas vinculadas con dichos aprendizajes. Esta persistencia, pese a las adaptaciones realizadas y a las "oportunidades" 
coyunturales ofrecidas por el nuevo contexto, sugiere que se trata de un asunto de raigambre profunda en la formación de la carrera.

Entre los aprendizajes que deja el trabajo docente en pandemia, el equipo de cátedra puntualizó en aquellos vinculados con la adquisición de destrezas tecnológicas implicadas en la enseñanza virtual, la producción de material pedagógico con base en ese formato y otros referidos a los modos de relacionarse y establecer el encuadre en la comunicación no presencial con estudiantes. En este sentido, algunos auxiliares de docencia afirman:

\footnotetext{
aprendí a adaptarme a instrumentos que conocía parcialmente, especialmente plataformas para reuniones virtuales. En términos de mi persona, cambié ciertos estilos para dictar clases. (Auxiliar de docencia, comunicación personal, 15 de noviembre de 2020)

Percibí cuánto de mi posibilidad de compartir ciertos contenidos con los/as estudiantes descansaba en la interacción, el tono de la voz, la gestualidad, la posibilidad de preguntar y dialogar en clases. (Auxiliar de docencia, comunicación personal, 16 de noviembre de 2020)
}

Como puede leerse en las respuestas docentes, el escenario impulsó modificaciones relevantes en su tarea cotidiana al demandar nuevos aprendizajes y actitudes para establecer el vínculo pedagógico. La redefinición del encuadre, aunque fuera señalada como fuente de ansiedades y mayores cargas laborales, también fue considerada como un estímulo para poner en juego habilidades poco exploradas o adquirir destrezas que resultaban ajenas hasta el momento. De manera más evidente que en las respuestas de estudiantes, se reiteró entre docentes que la virtualidad exigió mayor pericia académica, pedagógica e informática. Además, vimos interpelada nuestra situación en tanto trabajadores y trabajadoras que sufrieron de manera intempestiva la modificación de las rutinas laborales, cambio simultáneo a las nuevas dinámicas y exigencias del entorno hogareño (Scholten et al., 2020).

\section{Conclusiones}

El dictado de la materia Problemática de la Salud en modalidad virtual durante la pandemia y el ASPO supuso múltiples cambios respecto de las experiencias previas en la docencia universitaria. Nos incitó a revisar inercias y falencias en las formas de enseñar que veníamos desarrollando. También nos posibilitó descubrir nuevas formas de encarar la tarea y los vínculos entre docentes de la materia, con estudiantes y con otras asignaturas del plan de estudios de la carrera de Trabajo Social.

La abrupta mudanza a la enseñanza virtual fue una estrategia impuesta por el contexto, en un período muy breve, sin las adaptaciones pedagógicas, materia- 
les y tecnológicas que requería un proceso de tal magnitud. Para una parte del equipo docente, la experiencia tuvo más que ver con continuar educando en la emergencia que con explorar ciertos alcances de la virtualidad como estrategia pedagógica. Sin embargo, creemos que la pandemia y el ASPO propiciaron un entorno de "mayor ubicuidad" en el desarrollo de la materia, que si bien no estuvo propuesto como tal por nosotras, posibilitó aprendizajes constantes en nuevos escenarios y en distintos momentos. Los procesos educativos se integraron a la cotidianidad de los y las participantes de la experiencia y requirieron — por parte de los/as docentes - amoldar contenidos y estrategias pedagógicas a las características de los entornos sociales, comunicativos y vitales tanto de las y los estudiantes como propios.

Aun en el contexto adverso, docentes y estudiantes destacaron que la modalidad de dictado virtual realizada por la cátedra permitió sostener el proceso de enseñanza-aprendizaje, en un escenario sociosanitario crítico e incierto. Para unas y otros, no obstante, las condiciones contextuales funcionaron como límites, presiones y sobrecargas. Entre estudiantes, las condiciones de vida, hogareñas, las dificultades tecnológicas y las cargas de cuidado aparecían como limitaciones para sostener el cursado virtual. Entre docentes, las limitaciones fueron similares, pero se adicionaron la mayor dedicación horaria que requirió el dictado virtual, el avance sobre el tiempo de descanso y las nuevas exigencias tecnológicas para realizar las tareas habituales.

En cuanto a las ventajas del desarrollo virtual ponderadas por cada uno de estos grupos, entre estudiantes se destacó la mayor autonomía en la gestión de los horarios y el ahorro de tiempo y dinero por no tener que trasladarse hasta la universidad. Entre docentes, la horizontalidad y fluidez en los vínculos con estudiantes, junto a la mayor calidad y dedicación en los materiales pedagógicos, se destacan como valoraciones positivas de la virtualidad. En este sentido, pensamos que tener que producir contenidos de manera más amigable, recuperar saberes que se despliegan en los primeros años de la carrera y generar adecuaciones creativas para sostener la enseñanza en tiempos de pandemia y de ASPO nos compelió a los y las docentes a mejorar la calidad de los intercambios hacia adentro y puertas afuera de la cátedra.

Es menester leer estas percepciones en el marco del contexto social que las albergó. Algunos de los aspectos positivos del proceso aludido descansaron, en gran parte, en elementos excepcionales vinculados con la pandemia de coronavirus y las medidas de aislamiento. Con ello queremos destacar que la dedicación docente, muy por encima de los horarios acordados y los salarios percibidos, fue el resultado de un compromiso asumido —especialmente con la educación pública y el estudiantado— para afrontar una situación inédita. Sin 
embargo, consideramos que las estrategias de educación virtual y los aportes que pueden realizar para revisar la formación universitaria no deben sostenerse en la sobrecarga de los educadores y las educadoras. Por esta razón, un debate complejo que deberemos encarar es el modo en que se desplegará la virtualidad en la educación universitaria durante los próximos años.

\section{Agradecimientos}

Agradecemos a la profesora María José López por la lectura atenta del artículo y las sugerencias realizadas.

\section{Referencias}

Boni Aristizábal, A., Lozano Aguilar, J. y Walker, M. (2010). La educación superior desde el enfoque de capacidades. Una propuesta para el debate. Revista Electrónica Interuniversitaria de Formación del Profesorado, 13(3), 123-131. https://dialnet.unirioja.es/servlet/articulo?codigo=3307562

Burbules, N. (2014) El aprendizaje ubicuo: Nuevos Contextos, Nuevos Procesos Revista Entramados: educación y sociedad, 1(1), 131-134. https://dialnet.unirioja.es/servlet/articulo?codigo $=5251816$

Díez-Gutiérrez, E. y Díaz-Nafría, J. (2018). Ecologías de aprendizaje ubicuo para la ciberciudadanía crítica. Comunicar, 26(54), 49-58. https://doi.org/10.3916/C54-2018-05

Dirección de Economía, Igualdad y Género (2020). Los cuidados, un sector económico estratégico. Medición del aporte del Trabajo doméstico y de cuidados no remunerado al Producto Interno Bruto. Ministerio de Economía.

Evans, R., Barer, M. y Marmor, T. (eds.). (1996). ¿Por qué alguna gente está sana y otra no? Los determinantes de salud de las poblaciones. Díaz de Santos.

Expósito, E. y Marsollier, R. (2020). Virtualidad y educación en tiempos de COVID-19. Un estudio empírico en Argentina. Educación y Humanismo, 22(39), 1-22. https://doi.org/10.17081/eduhum.22.39.4214

Fueyo Gutiérrez, A; Braga Blanco, G.; Fano Méndez, S. (2015). Redes sociales y educación: el análisis socio-político como asignatura pendiente. Revista Interuniversitaria de Formación del Profesorado, 29 (1), pp. 119-129. https://www.redalyc.org/articulo.oa?id=27439665009

García, MH., Miota, A., Barranco Tirado, J.; Belda Grindley, C., Páez Muñoz, E., Rodríguez Gómez, S., Lafuente Robles, N. (2019). Guía breve. Salud Comunitaria Basada en activos. Granada. Escuela Andaluza de Salud Pública. https://www.easp.es/wp-content/uploads/dlm_uploads/2019/01/ EASP_Guia_breve_SaludComActivos-FUM-05-12-18.pdf

Jara, O. H. (2013). La sistematización de experiencias. Para otros mundos posibles. Instituto Mexicano para el Desarrollo Comunitario (Imdec).

Lobos, N. (2020). Ni un solo milagrito. Sobre la recepción de las teorías críticas en Trabajo Social. Revista Margen, (98), 1-12. https://www.margen.org/suscri/margen98/Lobos-98.pdf

López Arellano, O., Escudero, J. C. y Dary Carmona, L. (2008). Los determinantes sociales de la salud: una perspectiva desde el Taller Latinoamericano de Determinantes Sociales de la Salud, ALAMES. Medicina Social 3(4). 323-335. https://www.medicinasocial.info/index.php/medicinasocial/article/ view/260/538 
Martínez-Garcés, J. y Garcés-Fuenmayor, J. (2020). Competencias digitales docentes y el reto de la educación virtual derivado de la covid-19. Educación y Humanismo, 22(39), 1-16. https://doi. org/10.17081/eduhum.22.39.4114

Menéndez, E. (1990). El modelo médico hegemónico. Estructura, función y crisis. En E. Menéndez, Morir de alcohol. Saber y hegemonía médica (pp. 83-117). Alianza editorial mexicana.

Molina Jaramillo, A. (2018). Territorio, lugares y salud: redimensionar lo espacial en salud pública Cadernos de Saúde Pública 34(1), e00075117. http://dx.doi.org/10.1590/0102-311X00075117

Merhy, E., Camargo, L., Feuerwerker, M. y Burg, R. (2006). Educación Permanente en Salud: una Estrategia para Intervenir en la Micropolítica del Trabajo en Salud. Salud Colectiva, 2(2), 147-160. https://doi.org/10.18294/sc.2006.62

Minayo, M. C. S. (2009). La artesanía de la investigación cualitativa. Lugar Editorial.

OMS/OPS (2017). Determinantes sociales de la salud en la región de las Américas https://www.paho. org/salud-en-las-americas-2017/

OMS (2008). Determinantes sociales de la salud. https://www.who.int/social_determinants/es/

OMS (1986). Carta de Ottawa para la Promoción de la Salud. I Conferencia Internacional sobre la Promoción de la Salud. https://bit.ly/3iMtukR

OMS/OPS (1986). Promoción de la salud. https://www.paho.org/es/temas/promocion-salud

Petracci, M. (2012). Comunicación y Salud en la Argentina. LCRJ Arero

Pría Barros, M. C. (2006) Análisis de la situación de salud en áreas pequeñas. En S. Martínez Calvo, M. Caraballoso Hernández, M. Astraín Rodríguez, M. Pría Barros, V. Perdomo, C. Arocha Mariño, et al. (eds.), Análisis de la situación de salud (pp. 105-122). Editorial Ciencias Médicas.

Ramos, S. (2006). La sociología y la medicina: por qué, para qué y cómo construir puentes. Nuevos documentos Cedes. https://repositorio.cedes.org/handle/123456789/3613

Scholten, H., Quezada-Scholzb, V., Salas, G., Barria-Asenjo, N., Rojas-Jara,C., Molina, J., García, M.T., Jorquera, J., Marinero Heredia, A., Zambrano, A., Gómez Muzzio, E., Cheroni Felitto, A., CaychoRodríguez, T., Reyes-Gallardo,T., Pinochet Mendoza, N., Bindeo, P., Uribe Muñoz, J., Bernal Estupiñan, J. y Somarrivae, F. (2020). Abordaje psicológico del Covid-19: una revisión narrativa de la experiencia latinoamericana. Revista Interamericana de Psicología, 54(1). https://www.preventionweb.net/files/72089_abordajepsicologicodelcovid.pdf

Schord Landman, C. (2020). Consecuencias subjetivas de la pandemia. Pensar la pandemia. Documento de trabajo Clacso, (18). https://www.clacso.org/consecuencias-subjetivas-de-la-pandemia/

Ståhl, T., Wismar, M., Ollila, E., Lahtinen E. y Leppo, K. (eds). (2006). Health in All Policies. Prospects and potentials. Finland Ministry of Social Affairs and Health and European Observatory on Health Systems and Policies.

Volman G., Raimondi C., Maturano K. (2020). Trayectorias académicas atravesadas por la pandemia. Documento elaborado por el Equipo de Orientación Sapoe-Traces, en el marco de la Secretaría Académica de la Facultad de Ciencias Políticas y Sociales de la Universidad Nacional de Cuyo. 